\title{
Electrocardiogram Characteristics of Different Motor Types of Parkinson's Disease
}

This article was published in the following Dove Press journal:

International Journal of General Medicine

\author{
Ling-Ling Zhong' \\ Ya-Qi Song ${ }^{2}$ \\ Ke-Ju Ju' \\ Ai-Nian Chen' \\ Hua Cao' \\ 'Department of Neurology, The Affiliated \\ Huaian No. I People's Hospital of Nanjing \\ Medical University, Huai'an, Jiangsu, \\ People's Republic of China; ${ }^{2}$ Department \\ of Radiation Oncology, The Affiliated \\ Huaian No. I People's Hospital of Nanjing \\ Medical University, Huai'an, Jiangsu, \\ People's Republic of China
}

Correspondence: Hua Cao

Department of Neurology, The Affiliated Huaian No. I People's Hospital of Nanjing Medical University, I Huanghe Road

West, Huai'an, 223300, Jiangsu, People's

Republic of China

Tel +86 I390I405828

Fax +86 517-84907287

Email caohua_hh@163.com

\begin{abstract}
Aim: This study aims to investigate the electrocardiogram characteristics of the different motor types of Parkinson's disease.

Methods: The data on 118 patients with Parkinson's disease (PD), who were initially diagnosed in the Outpatient and Inpatient Department, was collected. Among these 118 PD patients, 74 patients were assigned to the PIGD group, while 44 patients were assigned to the TD group, and their clinical features were analyzed, which included age, course, disease classification, and electrocardiogram parameters (PR, QRS, QT interval, and QTC).

Results: The QT interval in PD patients was positively correlated with the course of the disease and Hoehn-Yahr stage, and the QT interval in the PIGD group was longer than that in the TD group.
\end{abstract}

Conclusion: A prolonged QT interval may indicate a longer disease period and a more severe disease condition.

Keywords: Parkinson's disease, autonomic nervous function, symptom, electrocardiogram, ECG, QT interval

\section{Introduction}

Parkinson's disease (PD) is a degenerative disease of the nervous system, the main manifestations of which include both motor and non-motor symptoms. Among these non-physical symptoms, the incidence of autonomic nerve function symptoms is higher, such as cardiovascular, gastrointestinal, genitourinary tract, temperature regulation, or pupil dysfunction. ${ }^{1}$ As the incidence and morbidity of PD continuously increase, the dysfunction of the autonomic nervous system will increase the social and economic burden. Furthermore, there have been many reports that the cardiovascular autonomic dysfunction of patients with PD exhibited a decrease in heart rate. ${ }^{2}$ The previous studies have shown that a decrease in heart rate would take place before the main symptoms of PD occur. ${ }^{3}$ In addition, the neural terminal degeneration of PD autonomic nerves would cause an interval extension in the QT interval. ${ }^{3}$ However, many drugs for PD treatment can also cause the extension of QT intervals. $^{4}$ In the present study, the clinical data of patients with PD at first diagnosis in the Department of Neurology of Huai'an First People's Hospital from January 2011 to December 2016 was collected, and a cross-sectional study was carried out to investigate the electrocardiogram features of these PD patients and patients with different physical types of PD, as well as the relevance between the electrocardiogram parameters and history of diseases. 


\section{Data and Methodology}

\section{Subjects of Research}

The data for patients with PD at first diagnosis, which was performed at the Department of Neurology of Huai'an First People's Hospital from January 2011 to December 2016, was collected. These patients did not receive anti-PD drugs before admission and did not use drugs that could affect their cardiac rates, such as antidepression drugs or atypical antipsychotic drugs.

The selection criteria for patients with PD were as follows: (1) patients who conformed to the diagnostic criteria for PD of the United Kingdom Parkinson's Disease Society Brain Bank; (2) patients who provided a signed informed consent, had no obvious cognitive dysfunction and were able to complete the evaluation scale independently; (3) patients classified by the Hoehn-Yahr classification: stage 1.0-2.0, early phase; stage $2.5-3.0$, middle phase; stage 4.0-5.0, late phase. Exclusion criteria: (1) patients with dementia or significant memory disorder (Mini-Mental State Examination [MMSE], a score < 24); (2) PD patients with syndromes caused by cerebrovascular diseases, poisoning or drugs, PD patients with additional comprehensive syndromes, such as patients with combined heart disease, and PD patients who received drugs that could affect their heart rates. This study was conducted with approval from the Ethics Committee of the Affiliated Huaian No. 1 People's Hospital of Nanjing Medical University. This study was conducted in accordance with the declaration of Helsinki. Written informed consent was obtained from all participants. The study is in compliance with the COPE guidelines. No medication for Parkinson's disease was used before admission, and no medication that affected heart rate such as antidepressants, antidepressants, or atypical antipsychotics was used.

\section{Methods of Research}

1) Electrocardiogram: The Japanese Fukuda synchronous 12-lead electrocardiogram machine is used to record the interval phase of the QRS, QTC, and QT of patients, and calculate the QTC. QTC $=\mathrm{QT} /(\mathrm{RR} \wedge 0.33) \mathrm{RR}$ is the standard value of a heart rate, which can be obtained by dividing 60 by the heart rate.

2) For disease seriousness, the physical subtype application of the Hoehn-Yahr stage was performed to evaluate the stages of PD. Hoehn-Yahr staging: stage 1.0-2.0, early phase; stage 2.5-3.0, middle phase; stage 4.0-5.0, late phase. $^{5,6}$
3) For the statistical methods, SPSS 17.0 software was used for analysis, counting data were expressed as mean \pm standard deviation, inter-group measurement data was analyzed by $t$-tests, inter-group counting data was analyzed by $X^{2}$ tests, demographic data and clinical variables were used to determine the Spearman level correlation coefficient, a nonparametric Mann-Whitney $U$-test was performed to compare partially distributed measurement data, and $P<0.05$ was considered statistically significant. The questionnaire survey was conducted by the neurologist at or above the attending level and conducted on the first day of admission or the day of physical examination. The subjects and their families participated in the survey process.

\section{Results}

\section{General Situation of the Research Subjects}

Data for a total of 140 PD patients was initially collected. Based on the above criteria, 118 PD patients were selected for the present study. Among these patients, 66 patients were male $(55.93 \%)$. Their age range was $60-80$ years, with an average age of $69.09 \pm 0.78$ years, and the duration of the disease ranged from one month to five years. According to the Hoehn-Yahr classification $(2.90 \pm 0.09), 44$ patients were assigned to the TD group, while 74 patients were assigned to the PIGD group. The electrocardiogram PR interval, QRS, QTC, and QT interval were evaluated, and the result of Lead II showed statistical differences.

\section{Correlation Analysis of the PR Interval, QRS, QTC, and QT Interval, Age, Hoehn-Yahr Classification, and Disease \\ Course}

The PR interval is correlated to the Hoehn-Yahr classification and disease course $(\mathrm{r}=0.72, P<0.01 ; \mathrm{r}=0.72, P<$ $0.001)$, the QRS is correlated to Hoehn-Yahr classification and the disease course $(\mathrm{r}=0.82, P<0.01 ; \mathrm{r}=0.43, P<$ 0.01 ), QTC is correlated to age, Hoehn-Yahr classification, and disease duration $(\mathrm{r}=0.21, P<0.05 ; \mathrm{r}=0.76, P<0.001$, $\mathrm{r}=0.75 ; P<0.01)$, and QT interval is correlated to QTC and age, Hoehn-Yahr classification, and disease course $(\mathrm{r}=0.42$, $P<0.01 ; \mathrm{r}=0.77, P<0.001 ; \mathrm{r}=0.55, P<0.01$ ) (Table 1).

\section{Analysis of PR and QT Intervals in Various Physical Types of PD Patients}

There was no significant difference in age between the PIGD group and TD group $(\mathrm{t}=0.01, \mathrm{Pp}=0.91)$. 
Table I Correlation Analysis of QT, PR Interval, Age and H-Y Stages

\begin{tabular}{|l|l|l|l|l|l|l|l|}
\hline & Age (Year) & H-Y & Course of the Disease & P-R & QRS & QTC & QT \\
\hline Age & $\mathrm{I}$ & 0.026 & $0.25^{* *}$ & 0.05 & 0.1 & $0.21^{*}$ & $0.42^{* * *}$ \\
H-Y & 0.03 & $\mathrm{I}$ & $0.48^{* * *}$ & $0.72^{* *}$ & $0.82^{* *}$ & $0.76^{* * *}$ & $0.77^{* *}$ \\
Course of the disease (month) & $0.25^{* *}$ & $0.48^{* * *}$ & $\mathrm{I}$ & $0.35^{* *}$ & $0.43^{* *}$ & $0.74^{* * *}$ & $0.55^{* *}$ \\
PR (seconds) & 0.05 & $0.72^{* * *}$ & $0.35^{* * *}$ & 1 & $0.71^{* *}$ & $0.56^{* * *}$ & $0.58^{* *}$ \\
QRS (seconds) & 0.1 & $0.82^{* * *}$ & $0.43^{* * *}$ & $0.7 I^{* *}$ & $\mathrm{I}$ & $0.7 I^{* * *}$ & $0.72^{* *}$ \\
QTC & $0.21^{*}$ & $0.76^{* * *}$ & $0.75^{* * *}$ & $0.56^{* *}$ & $0.7 I^{* *}$ & $\mathrm{I}$ & $0.79^{* *}$ \\
QT (seconds) & $0.42^{* *}$ & $0.77^{* * *}$ & $0.55^{* * *}$ & $0.58^{* *}$ & $0.72^{* *}$ & $0.79^{* * *}$ & $\mathrm{I}$ \\
\hline
\end{tabular}

Notes: $* \mathrm{P}<0.05, * * \mathrm{P}<0.01, * * * \mathrm{P}<0.001$.

Patients in the PIGD group had a longer duration disease course than patients in the TD group $(\mathrm{t}=5.30$, $P<0.0001)$. Furthermore, the Hoehn-Yahr classification was higher in the PIGD group than in the TD group $(\mathrm{t}=$ $7.51, P<0.0001)$, the PR interval was longer in the PIGD group than in the TD group $(\mathrm{t}=4.80, P<0.0001)$, the wavelength of QRS was longer in the PIGD group than in the TD group $(\mathrm{t}=5.18, P<0.0001)$, and the interval of QT was longer in the PIGD group than in the TD group ( $\mathrm{t}=2.345, P=0.02$ ) (Table 2). Correlation of UPDRS with ECG is shown in Table 3.

\section{Discussion}

Patients with PD have an increased risk of heart disease during the course of the disease. This may develop into autonomic nerve dysfunction, cardiomyopathy, coronary heart disease, arrhythmias, or sudden cardiac death, and the most prominent is the abnormality of autonomic nerve function. ${ }^{7}$ At present, the examination items of cardiac function for PD patients include individual and family disease history, clinical examination, blood pressure measurement, electrocardiogram, X-ray, echocardiography, blood pressure, and MIBG SPECT (scintillation method). At present, heart symptoms of PD have gradually attracted attention. However, few studies have been conducted on the electrocardiogram features of PD and various physical PD types. Many drugs for PD treatment can also cause the extension of QT intervals. ${ }^{4}$ In the present study, the clinical data of patients with PD at first diagnosis in the Department of Neurology of Huai'an First People's Hospital from January 2011 to December 2016 was collected, and a cross-sectional study was carried out to investigate the electrocardiogram features of these PD patients and patients with different physical types of PD, as well as the relevance between the electrocardiogram parameters and history of diseases.

Since its early description, researchers have realized that PD is correlated to autonomous dysfunction and that autonomic nerve dysfunction is common. Hence, it can also present as cardiac autonomic nerve dysfunction. ${ }^{7}$ The autonomic dysfunction of the heart of PD patients may be the result of the degeneration of the sympathetic and parasympathetic neurons. ${ }^{8}$ Previous studies have reported that the PR interval is correlated to autonomic nerve dysfunction in patients. Foreign scholars have studied 191 patients with PD and found that results of MIBG scintigraphy in 19 PR patients were abnormal. ${ }^{9}$ Meanwhile, in the present study, it was found that PR is correlated to the Hoehn-Yahr classification. It was further speculated that the prolongation of PR could be used as a biomarker for autonomic nerve dysfunction in PD. In addition, the present study was consistent with the scholars' reports. ${ }^{9}$ In the present study, it was found that the QT interval was positively correlated with the disease course and Hoehn-Yahr

Table 2 PR and QT Interval Analysis of PD Groups with Different Motor Types

\begin{tabular}{|l|l|l|l|l|}
\hline Project & TD & PIGD & t & P \\
\hline Age (year) & $68.98 \pm 1.14$ & $69.16 \pm 1.04$ & $\mathrm{t}=0.1 \mathrm{I}$ & $0.9 \mathrm{I}$ \\
Course of the disease (month) & $6.86 \pm 1.14$ & $19.58 \pm 1.72$ & $\mathrm{t}=5.30$ & $<0.000 \mathrm{I}$ \\
H-Y & $2.15 \pm 0.10$ & $3.35 \pm 0.11$ & $\mathrm{t}=7.5 \mathrm{I}$ & $<0.000 \mathrm{I}$ \\
PR (seconds) & $0.15 \pm 0.002$ & $0.16 \pm 0.002$ & $\mathrm{t}=4.80$ & $<0.000 \mathrm{I}$ \\
QRS (seconds) & $0.064 \pm 0.0007$ & $0.092 \pm 0.004$ & $\mathrm{t}=5.18$ & \\
QT (seconds) & $0.34 \pm 0.004$ & $0.41 \pm 0.006$ & $\mathrm{t}=2.345$ & $0.000 \mathrm{I}$ \\
\hline
\end{tabular}

Note: Sample size: a total of I40 PD patients. 
Table 3 Correlation of UPDRS with ECG

\begin{tabular}{|l|l|l|l|l|}
\hline & PR & QRS & QTC & QT \\
\hline UPDRS & 0.017 & 0.18 & 0.195 & 0.23 \\
\hline
\end{tabular}

classification. The QT interval represents the total time course of ventricular depolarization and repolarization. In pathological studies of PD, it has found that cardiac sympathetic nerve disorders affect the conduction system of the heart, including inter-node bundles, atrial bundles, and Purkinje fibers. ${ }^{10}$ With the development of the disease, sympathetic nerve dysfunction may lead to the extension of the QT interval of PD patients. In a large-scale study of normal subjects, the electrocardiogram analysis revealed that the QT interval increased with age, but no statistical analysis was performed. ${ }^{11,12}$ These present results revealed the positive correlation between QT and the age of PD patients. In summary, these findings suggest that the QT interval in elderly PD patients may be particularly long. In the past decade, researchers have given significant attention to PD mortality. In a large-scale cohort study, ten of $131 \mathrm{PD}$ patients (7.6\%) suffered sudden cardiac death. ${ }^{13}$ The extension of the QT interval would increase the risk of sudden cardiac death. ${ }^{14}$ In order to lower this risk, attention should be given to the QT interval in the treatment process of elderly PD patients.

In the present study, it was found that the disease course was longer and more serious in the PIGD group than in the TD group, and the PR intervals were longer in the PIGD group than in the TD group $(\mathrm{t}=4.80, P<0.0001)$. Furthermore, the wavelength of QRS was longer in the PIGD group than in the TD group $(\mathrm{t}=5.18, P<0.0001)$, and the QT intervals were longer in the PIGD group than in the TD group $(\mathrm{t}=2.345, P=0.02)$. Foreign scholars have revealed that a score of PIGD UPDRS III is higher than that in the TD group. Therefore, patients often present with more axial damage, and the dopamine treatment is usually poor. ${ }^{15}$ Through the comparison of the two subtypes, the loss of the substantia nigra region in TD patients, the vagus dorsal nucleus, and the dopamine neurons were fewer than those in the PIGD type, and the deposition of the cortical Lewy body in TD patients was less than that of the PIGD type. Through functional imaging studies, it was found that with the progression of the disease, the dopamine and glucose metabolism of the PIGD tail nucleus and the anterior part of the shell nucleus significantly decreased, while the TD type was relatively stable Therefore, compared with the TD type, the PIGD type has a poorer prognosis, with more abnormal gaits, as well as more non-physical symptoms. ${ }^{16-19}$ When the duration of disease was comparable between these groups, it was found that 123I-MIBG uptake was lower in patients with physical stiffness or unstable gait, suggesting a higher degree of myocardial sympathetic nerve dominance. ${ }^{20}$

However, we should note that the PR interval, QRS interval and QT interval in PD patients did not deviate much from normal values, indicating that they may not be significant enough for physicians to pay attention to them. In addition, the sample size of the current study is not large enough. Future studies need to include more patients and to figure out the exact changes of ECG characteristics, not only the ones we explored in this study but also other ECG parameters. This study also lacked a control group. These limitations should be avoided in future studies. Also, correlation analysis are not age-justified, so there is a missed opportunity by not applying logistic regression to classify TD vs PIGD by using age, gender, ECG characteristics etc as independent variables.

\section{Conclusion}

In summary, the clinical characteristics and the electrocardiogram parameters may be closely correlated. Several electrocardiogram parameters may reflect the progression of functional dysfunction or disease in the body. When treating PD patients, clinicians can refer to the parameters of the electrocardiogram, which may provide information for the disease. However, further studies are needed to confirm our findings.

\section{Data Sharing Statement}

We declared that materials described in the manuscript, including all relevant raw data, will be freely available to any scientist wishing to use them for non-commercial purposes, without breaching participant confidentiality. It can be obtained from the corresponding author.

\section{Ethics Approval and Consent to Participate}

I confirm that I have read the Editorial Policy pages. This study was conducted with approval from the Ethics Committee of the Affiliated Huaian No. 1 People's Hospital of Nanjing Medical University. This study was conducted in accordance with the declaration of Helsinki. 
Written informed consent was obtained from all participants.

\section{Acknowledgments}

We are particularly grateful to all the people who have given us help on our article.

\section{Disclosure}

The authors declare that they have no competing interests.

\section{References}

1. Arnao V, Cinturino A, Valentino F, et al. In patient's with Parkinson disease, autonomic symptoms are frequent and associated with other non-motor symptoms. Clin Auton Res. 2015;25:301-307. doi:10.1007/ s10286-015-0306-x

2. Alonso A, Huang X, Mosley TH, Heiss G, Chen H. Heart rate variability and the risk of Parkinson disease: the atherosclerosis risk in communities study. Ann Neurol. 2015;77:877-883. doi:10.1002/ ana. 24393

3. Deguchi K, Sasaki I, Tsukaguchi M, et al. Abnormalities of ratecorrected QT intervals in Parkinson's disease-a comparison with multiple system atrophy and progressive supranuclear palsy. J Neurol Sci. 2002;199:31-37. doi:10.1016/S0022-510X(02)00079-5

4. Malek NM, Grosset KA, Stewart D, Macphee GJ, Grosset DG. Prescription of drugs with potential adverse effects on cardiac conduction in Parkinson's disease. Parkinsonism Relat Disord. 2013;19:586-589. doi:10.1016/j.parkreldis.2013.02.004

5. Forsaa EB, Larsen JP, Wentzel-Larsen T, Alves G. What predicts mortality in Parkinson disease?: a prospective population-based long-term study. Neurology. 2010;75:1270-1276. doi:10.1212/ WNL.0b013e3181f61311

6. Hoehn MM, Yahr MD. Parkinsonism: onset, progression, and mortality. 1967. Neurology. 2001;57:S11-26.

7. Garcia-Ruiz PJ, Chaudhuri KR, Martinez-Martin P. Non-motor symptoms of Parkinson's disease a review ... from the past. J Neurol Sci. 2014;338:30-33. doi:10.1016/j.jns.2014.01.002

8. Baldacci F, Vergallo A, Del Dotto $\mathrm{P}$, et al. RE response to: "Entacapone, Parkinson's disease, "functional adrenergic denervation", and Takotsubo syndrome". Parkinsonism Relat Disord. 2015;21:427. doi:10.1016/j.parkreldis.2014.11.010
9. Mochizuki H, Ebihara Y, Ugawa Y, et al. PR prolongation and cardiac 123I-MIBG uptake reduction in Parkinson's disease. Eur Neurol. 2015;74:107-111. doi:10.1159/000439022

10. Ghebremedhin E, Tredici KD, Langston JW, Braak H. Diminished tyrosine hydroxylase immunoreactivity in the cardiac conduction system and myocardium in Parkinson's dis- ease: an anatomical study. Acta Neuropathol. 2009;118:777-784. doi:10.1007/s00401009-0596-y

11. Rijnbeek PR, van Herpen G, Bots ML, et al. Normal values of the electrocardiogram for ages 16-90 years. $J$ Electrocard. 2014;47:914-921. doi:10.1016/j.jelectrocard.2014.07.022

12. Oka H, Mochio S, Sato H, Katayama K. Prolongation of QTc interval in patients with Parkinson's dis- ease. Eur Neurol. 1997;37:186-189. doi: $10.1159 / 000117432$

13. Sato K, Hatano T, Yamashiro K, et al.; Juntendo Parkinson Study Group. Prognosis of Parkinson's disease: time to stage III, IV, V, and to motor fluctuations. Mov Disord. 2006;21:1384-1395. doi:10.1002/ mds. 20993

14. Straus SM, Kors JA, De Bruin ML, et al. Prolonged QTc interval and risk of sudden car- diac death in a population of older adults. $J \mathrm{Am}$ Coll Cardiol. 2006;47:362-367. doi:10.1016/j.jacc.2005.08.067

15. Crosiers D, Pickut B, Theuns JD, et al. Non-motor symptoms in a Flanders- Belgian population of 215 Parkinson's disease patients as assessed by the non-motor symptoms questionnaire. $\mathrm{Am}$ J Neurodegener Dis. 2012;1:160-167.

16. Lolekha P, Wongwan P, Kulkantrakorn K. Association between serum uric acid and motor subtypes of Parkinson's disease. J Clin Neurosci. 2015;22:1264-12647. doi:10.1016/j.jocn.2015.02.015

17. Eggers C, Schwartz F, Pedrosa DJ, Kracht L, Timmermann L. Parkinson's disease subtypes show a specific link between dopaminergic and glucose metabolism in the striatum. PLoS One. 2014;9: e96629. doi:10.1371/journal.pone.0096629

18. Akbilgic O, Kamaleswaran R, Mohammed A, et al. Electrocardiographic changes predate Parkinson's disease onset. Sci Rep. 2020;10:11319. doi:10.1038/s41598-020-68241-6

19. Fujishiro H, Frigerio R, Burnett M, et al. Cardiac sympathetic denervation correlates with clinical and pathologic stages of Parkinson's disease. Mov Disord. 2008;23:1085-1092. doi:10.1002/mds.21989

20. Goldstein DS. Cardiac denervation in patients with Parkinson disease. Cleve Clin J Med. 2007;74:S91-4. doi:10.3949/ccjm.74. Suppl_1.S91
International Journal of General Medicine

\section{Publish your work in this journal}

The International Journal of General Medicine is an international, peer-reviewed open-access journal that focuses on general and internal medicine, pathogenesis, epidemiology, diagnosis, monitoring and treatment protocols. The journal is characterized by the rapid reporting of reviews, original research and clinical studies across all disease areas. The manuscript management system is completely online and includes a very quick and fair peer-review system, which is all easy to use. Visit http://www.dovepress.com/ testimonials.php to read real quotes from published authors. 